\title{
Comparison between Flow-Induced Alignment Behaviors of Poly(styrene-block-2-vinylpyridine)s and Poly(styrene-block-isoprene)s Solutions near ODT
}

\author{
Yoshiaki TAKAHASHI, ${ }^{1, \dagger}$ Masanori NARUSE, ${ }^{2}$ Yoshihiko AKAZAwA, ${ }^{2}$ \\ Atsushi TAKANO, ${ }^{2}$ and Yushu MATSUSHITA ${ }^{2}$ \\ ${ }^{1}$ Department of Molecular \& Materials Sciences, Kyushu University, 6-1 Kasugakoen, Kasuga 816-8580, Japan \\ ${ }^{2}$ Department of Applied Chemistry, Nagoya University, Furo-cho, Chikusa-ku, Nagoya 464-8603, Japan
}

(Received May 16, 2005; Accepted August 15, 2005; Published December 15, 2005)

\begin{abstract}
Flow-induced structure of lamellar forming poly(styrene- $d_{8}-b l o c k$-isoprene)s (DI) solutions under steady shear flow are examined by small angle neutron scattering and compared with those of poly(styrene- $d_{8}$ block-2-vinylpyridine)s (DP) solutions based on the experimentally determined interaction parameters, $(\chi N)_{\text {eff }}$. For DI solutions, it is observed that parallel and perpendicular alignments of lamellae coexist at low shear rates and the latter become dominant at high shear rates in the quiescent ordered states and disordered states very close to the quiescent order-disorder transition (ODT). At still lower concentration $C$ below the quiescent ODT, flow-induced ordering with perpendicular alignment is observed. For DP solutions, coexistence of parallel and perpendicular alignments are only observed at low shear rates for relatively high $C$ above the ODT, while only perpendicular alignment was observed at lower $C$ near the ODT. It is concluded that the degree of perpendicular alignment (ratio of sector averaged intensities) is primarily determined by $(\chi N)_{\text {eff }}$ and reduced shear rates. In addition, concentration and shear rate regions in which parallel alignment coexists become narrower for the DP diblock copolymers consist of components with almost the same viscosities. [DOI 10.1295/polymj.37.900]

KEY WORDS Poly(styrene-block-isoprene)s / Poly(styrene-block-2-vinylpyridine)s / FlowInduced Structure / Order Disorder Transition / Lamellar Structure / Small Angle Neutron Scattering /
\end{abstract}

Flow effects on the structure of diblock copolymers near the order-disorder transition (ODT) have been extensively studied especially for those assume lamellae structure. It was reported that the lamellar domains in the ordered states are oriented to a particular direction under oscillatory and steady shear flows, but the direction depends on samples and experimental conditions. ${ }^{1-7}$

Under "reciprocating steady shear" (with large amplitude, typically $500 \%),{ }^{3}$ and steady shear flows, ${ }^{6,7}$ so-called parallel and perpendicular orientations as illustrated in Figure 1 coexist. Here, the normals of lamellae are parallel to the shear flow, shear gradient, and vorticity direction in the transverse, parallel, and perpendicular alignments, respectively. Roughly speaking, the latter is more favored near the ODT and at the same condition the former is dominant at low shear rates, while the latter is dominant at high shear rates in the quiescent ordered states. In addition, flow-induced ordering and alignment of the structure are also reported even in the disordered states. These observations are qualitatively explained by a theory proposed by Fredrickson, ${ }^{8}$ in which the fluctuation effects and the difference in the viscosities of the component polymers in the diblock copolymers are taken

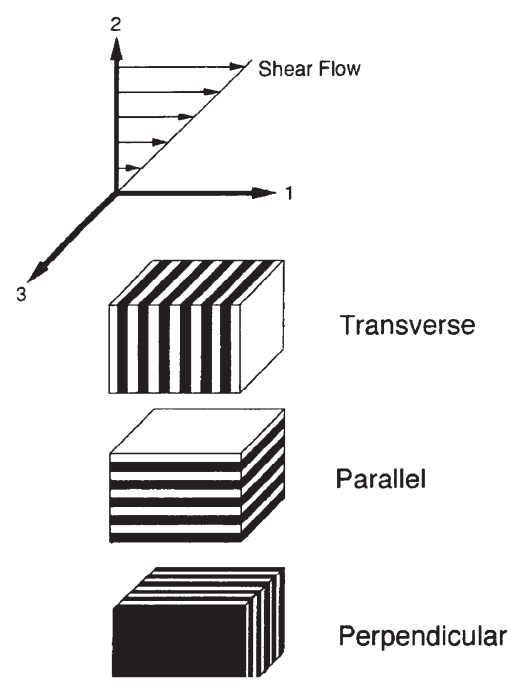

Figure 1. Schematic illustrations of the transverse, parallel, and perpendicular alignments of lamellae under shear flow. The normals of the lamellae are parallel to shear flow (1), shear gradient (2) and vorticity (3) directions in the transverse, parallel, and perpendicular alignment, respectively.

into account.

When lamellae structure is well aligned, the viscoelastic properties of block copolymers are dominated

${ }^{\dagger}$ To whom correspondence should be addressed (Tel/Fax: +81-92-583-8822, E-mail: ytak@mm.kyushu-u.ac.jp). 
by the responses from polymer chains since the contributions from large scale motions of grains become insensitive. ${ }^{1}$ Consequently, shear stress $\sigma$ and first normal stress difference $N_{1}$ become proportional to $\dot{\gamma}$ and $\dot{\gamma}^{2}$, respectively, where $\dot{\gamma}$ is shear rate. ${ }^{9}$

In previous papers, ${ }^{10-12}$ we studied the flow effects on lamellar structures of poly(styrene-block-2-vinylpyridine)s (SP) (and DP: S replaced by styrene- $d_{8}$ ) in a common good solvent, $\alpha$-chloronaphtharene $(\alpha \mathrm{CN})$, under steady shear flow by small angle neutron scattering and rheological measurements. The observed flow effects on the structure of SP and DP seems to be quite different from the data so far published as briefly described below.

For SP and DP diblocks, alignment of lamellae were observed only for relatively high molecular weight samples (higher than, say $350 \mathrm{k}$ ) in limited ranges of concentration and temperature near the ODT. ${ }^{10}$ The coexistence of parallel and perpendicular orientations are observed at low shear rates for relatively high concentration solutions, ${ }^{10}$ while only perpendicular alignment was observed for lower concentration samples. ${ }^{11}$ The $\dot{\gamma}$ region in which $N_{1}$ become proportional to $\dot{\gamma}^{2}$ was about two orders higher ${ }^{12}$ than that for poly(styrene-block-isoprene)s. ${ }^{9}$ When concentration was lowered very close to the ODT in the quiescent ordered states, such fine alignment of the structure was not observed. Further, a slight flow suppression of fluctuation effects but no flow-induced ordering was observed in the disordered states very close to ( $c a .0 .5 \%$ lower than) the ODT. ${ }^{12}$

One possible reason for above difference in flow induced structure of block copolymers is the difference in the interactions of components. Another possibility is the difference in viscoelastic properties of components, since two components of SP (and DP) have almost the same viscoelastic properties. ${ }^{13}$ In this paper, we study flow effects on the structure of poly(styrene$d_{8}$-block-isoprene)s (DI) solutions under steady shear flow by small angle neutron scattering (flow-SANS) at almost the same experimental conditions as used for DP diblock copolymers and compare the flowinduced structure of the both systems based on the experimentally determined interaction parameters.

\section{EXPERIMENTAL}

The diblock copolymer sample used was a poly(styrene- $d_{8}$-block-isoprene)s, DI-10, prepared by anionic polymerization method in vacuo. Weight-averaged molecular weight, $M_{\mathrm{w}}$, molecular weight distribution index, $M_{\mathrm{w}} / M_{\mathrm{n}}$, where $M_{\mathrm{n}}$ is number-averaged molecular weight, and volume fraction of styrene- $d_{8}, \phi_{\mathrm{PS}}$, of DI-10 are determined by combination of osmotic pressure, ${ }^{1} \mathrm{H}$ NMR and GPC measurements of the block copolymer and D chains remained after decomposition of I chain by ozonization, by the almost same methods as employed for similar block copolymers. ${ }^{14}$ The obtained values of $M_{\mathrm{w}}, M_{\mathrm{w}} / M_{\mathrm{n}}$, and $\phi_{\mathrm{PS}}$ are $1.15 \times 10^{5}, 1.12$, and 0.5 , respectively. A solvent used was dioctylphthalate (DOP).

Shear viscosities $\eta(\dot{\gamma})$ of DI-10 in DOP are measured by a Rheometrics mechanical spectrometer RMS800. A cone-plate geometry of $5 \mathrm{~cm}$ diameter and 0.04 rad cone-angle was used. Critical shear rates, $\dot{\gamma}^{*}$, at which the non-Newtonian behavior becomes apparent $\left(\eta\left(\dot{\gamma}^{*}\right) / \eta^{0}=0.8\right.$, where $\eta^{0}$ is zero shear viscosity) are obtained for 26 and $22 \%$ solutions. Zero shear viscosities of polystyrene and polyisoprene homopolymers corresponding to the respective block chains in DI-10 are also measured with RMS800. The polystyrene solutions had 2-3 times higher $\eta^{0}$ values than the polyisoprene solutions in the tested range of concentration (20-30 wt \%) at $27^{\circ} \mathrm{C}$.

Small angle neutron scattering (SANS) measurements were performed by SANS-U spectrometer at the Neutron Scattering Laboratory of the Institute for Solid State Physics, The University of Tokyo in Tokai, Ibaraki, Japan. The wavelength $\lambda$ and beam size of incident neutrons was $0.7 \mathrm{~nm}$ and $3 \mathrm{~mm} \phi$, respectively. The sample to detector distance was $8 \mathrm{~m}$. Ordinary quartz cells with sample thickness of $2 \mathrm{~mm}$ were used for determination of ODT condition. After the samples were set into the quartz cell, they were kept at $50{ }^{\circ} \mathrm{C}$ for one hour and cooled by $1^{\circ} \mathrm{C} / 5 \mathrm{~min}$ to room temperature to minimize the effects of flow and thermal histories. In addition to 10 solutions of DI-10, a few solutions of DP-20 $\left(M_{\mathrm{w}}=36.0 \times 10^{4}\right.$, $M_{\mathrm{w}} / M_{\mathrm{n}}=1.05$ and $\left.\phi_{\mathrm{PS}}=0.53\right)$ in $\alpha \mathrm{CN}$ at lower concentrations than those in a previous study ${ }^{15}$ are measured in this work to obtain the interaction parameter in a wide range of concentration.

Flow-SANS measurements were carried out by using a couette type flow cell ${ }^{16}$ attached to SANS-U. The gap between aluminum inner stator and quartz outer rotor was $0.75 \mathrm{~mm}$. Two scattering configurations, i.e., so-called through view, in which the incident neutron beam is in the direction normal to the surface of the center part of couette cell (along the shear gradient direction) and edge (tangential) view, in which the incident beam going through the edge of cell (along the shear flow direction) were employed. The latter was only measured for a short time (due to the restriction of beam time) to confirm the direction of alignments, especially for the existence or absence of parallel alignments of the lamellar structure.

The ordinary SANS data and the through view data are corrected for the incoherent scattering of the solvent and the empty cell. From the ordinary SANS data, circularly averaged intensity $I$ was obtained as 


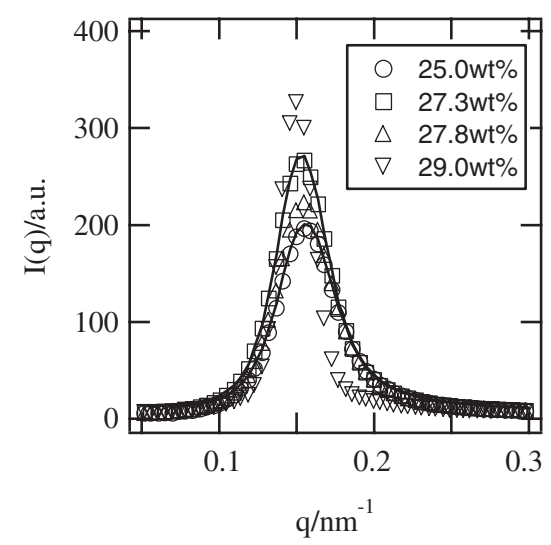

Figure 2. Plots of $I(q)$ vs. $q$ at different concentrations. Concentrations are denoted in the figure. Solid lines denote fitting results to the Leibler's theory.

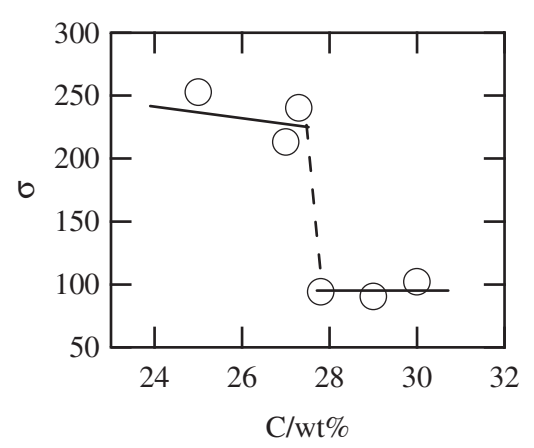

Figure 3. Concentration dependence of $\sigma$. Solid and broken lines are guide for eyes.

a function of the magnitude of wave vector, $q$, defined by $q=4 \pi \sin (\theta / 2) / \lambda$, where $\theta$ is scattering angle. From the flow-SANS data, sector averaged intensities within sectors of $\pm 5^{\circ}$ arc in horizontal $\left(I_{X}\right)$ and vertical $\left(I_{Y}\right)$ directions on the detector were obtained as a function of $q$. All the measurements are carried out at room temperature $\left(27^{\circ} \mathrm{C}\right)$.

\section{RESULTS AND DISCUSSIONS}

Figure 2 shows examples of plots of $I(q)$ against $q$ for DI-10 solutions. It is clear that $I(q)$ for $29.0 \%$ solution is narrower than other data, while other data are similar with each other. The peak intensities do not change in the order of concentration, reflecting the difference in degree of alignment of lamellae remained even after the annealing, so that the peak intensities cannot be used to determine the ODT. Here, we use square of full width at half maximum values, $\sigma$, of these scattering data to determine the ODT. ${ }^{17}$ Figure 3 shows plots of $\sigma v s$. C. It is clear that ODT of this sample is in between 27.8 and $27.3 \mathrm{wt} \%$ since $\sigma$ discontinuously changed by small change of $C$ in between these concentrations.

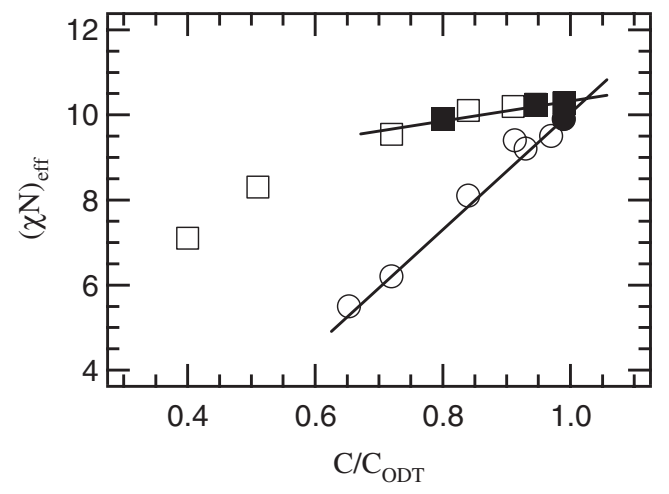

Figure 4. Plots of $(\chi N)_{\mathrm{eff}} v s . C / C_{\mathrm{ODT}}$ for DI-10 (square) and DP-20 (circle) solutions. Solid lines are guide for eyes. Filled symbols denote the concentration at which flow-SANS measurements are performed.

All data in the disordered states are fitted to the theoretical function of Leibler ${ }^{18}$ as shown by solid lines in Figure 2 by the same method as in a previous work $^{15}$ to obtain effective values of $\chi N,(\chi N)_{\text {eff }}$, where $\chi$ and $N$ are the Flory-Huggins interaction parameter and the number of statistical segments per copolymer molecules, respectively. The fitted values of $(\chi N)_{\text {eff }}$ may be different from the true values since corrections such as desmearing are not carried out in the analysis. However, the fitted theoretical curves well represent overall shape of scattered profiles as shown in Figure 2, in quite similar extent as those reported for DP-20 in the previous study, ${ }^{15}$ so that we use these experimentally obtained $(\chi N)_{\text {eff }}$ values to compare the two different systems.

Figure 4 shows plots of $(\chi N)_{\mathrm{eff}} v s . C / C_{\mathrm{ODT}}$ for DI10 and DP-20 solutions. Here, $C_{\mathrm{ODT}}(27.5 \%$ for DI- 10 and $12 \%$ for DP-20) is median value of the concentration range in which ODT is located. It is clear that the data for DI-10 very gradually decrease with decrease of $C$ near the ODT, while those for DP-20 almost linearly decrease with decrease of $C$. Thus, we conclude that the fluctuation effects are much stronger in DI systems than in DP systems, even both polymers have similar $C-M$ relations for the ODT. ${ }^{15,19}$

In the flow-SANS measurements, an attempt was made to erase the flow effects during sample setting as follows. The inner stator of couette cell was set at a position slightly higher than the measuring position and the temperature was kept at about $50{ }^{\circ} \mathrm{C}$ for $15 \mathrm{~min}$. Then the cell was gradually cooled to room temperature. At the same time, the stator was lowered to the measuring position very slowly to compensate the effects of volume change due to cooling. During this procedure, neutron scattering profile was monitored on the two dimensional detector. For $27.3 \%$ solution, the anisotropic profile observed at the beginning disappeared during the annealing. Figure 5a 
(a)

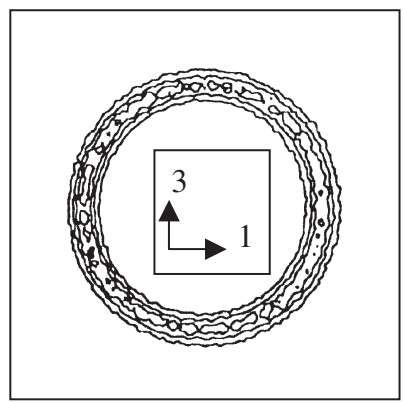

(b)

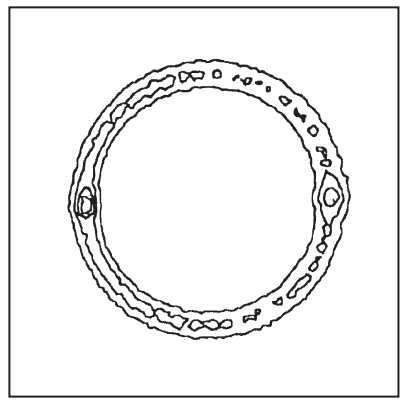

Figure 5. Contour maps of SANS in through view at quiescent for 27.3 (a) and $27.8 \%$ (b) DI-10 solutions. Allows denote (1) shear flow and (3) vorticity directions, respectively.

shows the isotropic scattering pattern for $27.3 \%$ solution obtained after the cooling. For $27.8 \%$ solution, weak anisotropic peaks observed in the horizontal direction on the detector did not disappear during the annealing. The weakly anisotropic scattering pattern obtained after the cooling is shown in Figure $5 \mathrm{~b}$.

When shear flow is applied to $27.8 \%$ solution, the weak peaks in the horizontal direction disappeared and strong anisotropic peaks are observed in the vertical direction denoting perpendicular orientation of lamellae. When shear flow is applied to 27.3 and $26.0 \%$ solutions, strong anisotropic peaks are also observed in the vertical direction as shown in Figure 6a, denoting the occurrence of flow-induced ordering and alignment of microdomains. On the other hand, only weak anisotropy is observed for $22 \%$ solution even at the highest shear rate, $100 \mathrm{~s}^{-1}$, which is about 4 orders higher than the shear rates used for other solutions.

Figure 7 shows examples of plots of $I_{\mathrm{X}}$ and $I_{\mathrm{Y}} v s . q$ for 27.3 and $22.0 \%$ solution at different shear rates. Here, $I_{\mathrm{X}}$ and $I_{\mathrm{Y}}$ reflect perpendicular and transverse alignments, respectively. For $27.3 \%$ solution, peak intensity of $I_{Y}$ increases with increase of shear rate, while that of $I_{\mathrm{X}}$ is almost constant at a value about one order lower than that without shear. For $22.0 \%$ solution, the peak intensity of $I_{\mathrm{X}}$ (not shown) is about $30 \%$ lower than that of $I_{Y}$ (circles) even at $100 \mathrm{~s}^{-1}$. From the difference in the shapes of these scattering data, it is clear that flow-induced microphase separation does not occur for $22.0 \%$ solution. The weak (a)

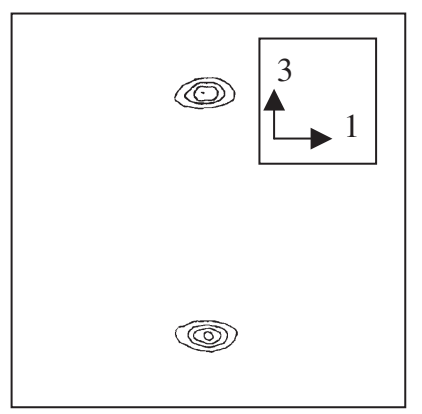

(b)

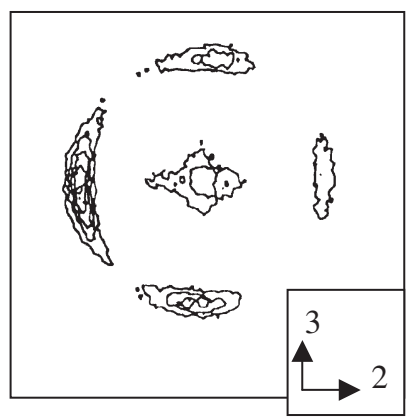

Figure 6. Contour maps of SANS in through view (a) and edge view (b) for the $27.3 \%$ solution. Shear rate is $0.04 \mathrm{~s}^{-1}$. Allows denote (1) shear flow, (2) shear gradient, and (3) vorticity directions, respectively.

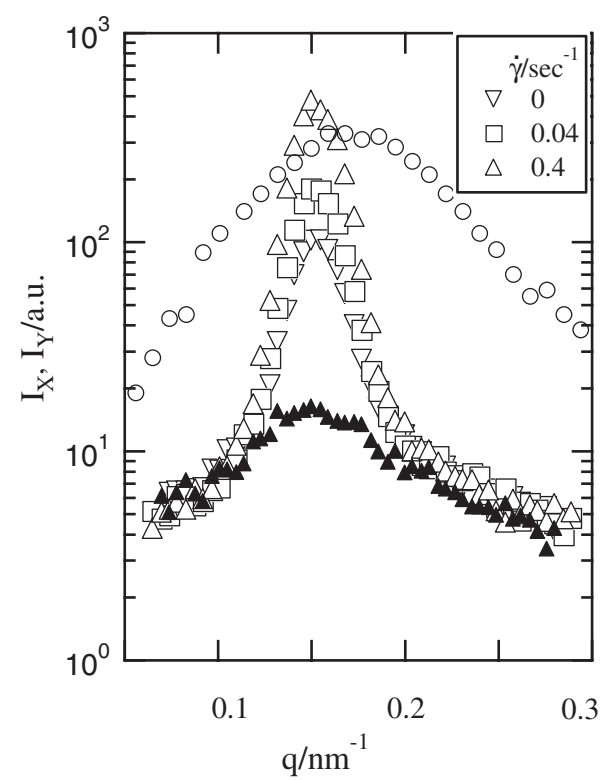

Figure 7. Plots of $I_{\mathrm{X}}$ and $I_{\mathrm{Y}} v s . q$ at different conditions. Filled symbols denote $I_{\mathrm{X}}$ data. Circles denote $I_{\mathrm{Y}}$ data for $22 \%$ solution at $100 \mathrm{~s}^{-1}$, while other symbols denote the data for $27.3 \%$ solution at different shear rates. The shear rates are denoted in the figure.

anisotropy observed for $22.0 \%$ solution immediately disappeared after the cessation of flow.

Figure $6 \mathrm{~b}$ shows an example of scattering pattern observed in the edge view observation. A streak in the horizontal direction located at around the center position of the detector is due to the scattering from 


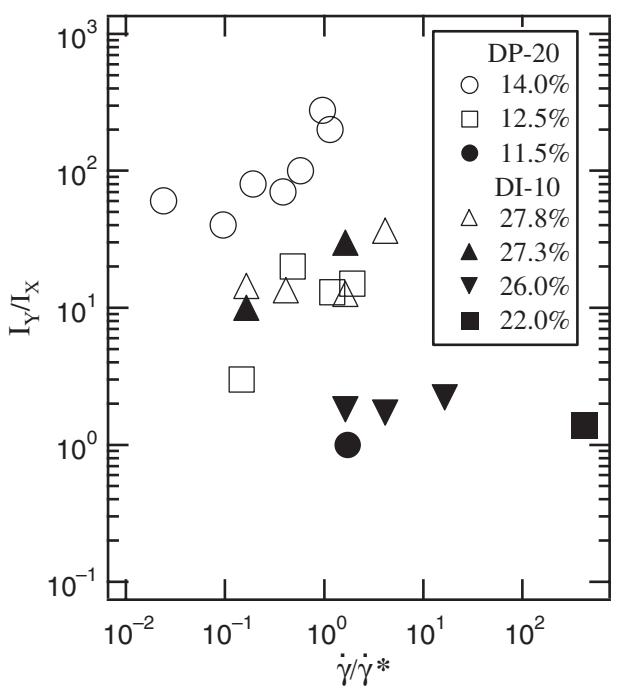

Figure 8. Plots of $I_{\mathrm{Y}} / I_{\mathrm{X}}$ vs. $\dot{\gamma} / \dot{\gamma}^{*}$ for DP-20 and DI-10 solutions. Symbols are denoted in the figure. Filled symbols denote the concentrations in the quiescent disordered states.

cell surface. The arc-shaped peaks in the vertical and horizontal directions reflect perpendicular and parallel alignments, respectively. It was observed that parallel alignments of lamellae (peaks in the horizontal direction) decrease while perpendicular alignments of them (peaks in the vertical direction) increase with increase of shear rate for 27.8 and $27.3 \%$ solutions. For $26 \%$ solution, on the other hand, the peak in the horizontal direction is not observed even at the lowest shear rate $\left(0.4 \mathrm{~s}^{-1}\right)$. These results are qualitatively the same as those reported by Winey et al. ${ }^{4,5}$

Figure 8 compares the $I_{\mathrm{Y}} / I_{\mathrm{X}}$ values for DI-10 and DP- $20^{11,12}$ as a function of reduced shear rate, $\dot{\gamma} / \dot{\gamma}^{*}$. It should be noted that $I_{\mathrm{X}}$ values are almost constant irrespective of shear rate and only slightly depends on concentrations, when the flow-induced alignment occurred. Experimentally obtained $\dot{\gamma}^{*}$ values are used for the all DP-20 solutions, ${ }^{12}$ while the $\dot{\gamma}^{*}$ value obtained for $26.0 \%$ solution of DI-10 is also used for solutions with slightly higher concentrations, 27.3 and $27.8 \%$, as an approximate value since it was very difficult to determine $\dot{\gamma}^{*}$ for these solutions.

It is clear that $I_{\mathrm{Y}} / I_{\mathrm{X}}$ values for $14.0 \%$ solution of DP-20 is higher than other data, denoting strong perpendicular alignment. The data for 27.3 and $27.8 \%$ solutions of DI-10 and $12.5 \%$ solution of DP-20 are similar with each other and they slightly increase with the increase of reduced shear rate. For $26.0 \%$ solution of DI-10, flow-induced microphase separation and perpendicular alignment of the lamellae are observed but $I_{\mathrm{Y}} / I_{\mathrm{X}}$ values are very small. For $22.0 \%$ DI-10 solution and $11.5 \%$ DI-20 solution, flow-induced microphase separation was not observed but a very slight anisotropic scattering pattern was observed for the latter.
According to Figure 4, it is apparent that 27.3 and $27.8 \%$ DI-10 solutions and 12.5\% DP-20 solution have quite similar $(\chi N)_{\text {eff }}$ values since these solutions are very close to the ODT for respective samples, while $14.0 \%$ DP-20 solution have the highest $(\chi N)_{\text {eff }}$ value among the tested solutions. It is also apparent that $22 \%$ DI-10 solution and $11.5 \%$ DI-20 solution have almost the same $(\chi N)_{\text {eff }}$ values. It is clear in Figure 8 that the solutions having similar $(\chi N)_{\text {eff }}$ values show similar behaviors. Thus, we conclude that the flow-induced alignment (and the flow-induced microphase separation) is primarily determined by interaction parameter and only perpendicular alignment is observed when quiescent disordered solution relatively far from the ODT undergoes flow-induced microphase separation, though there remain differences in the direction of alignments for different samples discussed later. The reason for the absence of flowinduced microphase separation phenomena for DP20 can be attributed to the steeper decrease of $(\chi N)_{\text {eff }}$ with the decrease of concentration or in other words, very narrow fluctuation regions.

As mentioned above, parallel and perpendicular alignments coexist for 27.3 and $27.8 \%$ DP-10 solutions, while only perpendicular alignment is observed for DP-20 even at the highest concentration. It should be noted that coexistence of parallel alignment was observed for $13.5 \%$ solution of DP-20 with a different experimental condition for flow-SANS at shear rates lower than $0.1 \mathrm{~s}^{-1}$, which is about one order lower than the lowest shear rate tested for $14.0 \%$ solution. ${ }^{6}$ Thus, it is concluded that the parallel alignment of lamellae is much stable for DI solutions than DP solutions compared at similar $(\chi N)_{\text {eff }}$ values and reduced shear rates.

The reduced shear rate employed in Figure 8 only reduces the difference in terminal relaxation time of block copolymer chains and it is different from the characteristic frequency employed in the theory of Fredrickson. ${ }^{8}$ However, comparison of the data at fixed $(\chi N)_{\text {eff }}$ values discussed above corresponds to the comparison at the same level of fluctuations. The viscosity ratio of components for DI-10 is $2-3$, while that of DP-20 is close to $1 .{ }^{13}$ Based on these experimental conditions, we can qualitatively compare with the theoretical predictions. We conclude that all the above results of flow-induced alignments are consistent with the theoretical predictions of Fredrickson; ${ }^{8}$ perpendicular alignment is dominant at high shear rates for diblock copolymers and become more stable at lower shear rates when the viscosity ratio of block components is close to 1 . The parallel alignment coexists only at low shear rates for high concentrations for the diblock copolymers with components' viscosity ratio close to 1 . Only perpendicular alignment is 
observed when flow-induced microphase separation occurs for a sample relatively far from ODT.

Finally, we briefly comment on the observations reported for other samples. ${ }^{3-7,10,12}$ For polyolefin block systems such as poly(ethyrenepropylene)-block-poly(ethylethylene), ${ }^{3}$ viscosity ratio of components are not known, however, at least the frequency dependence of alignments (parallel alignment at low frequency and perpendicular alignment at high frequency) is consistent with above discussions. For poly(styreneblock-isoprene)s, ${ }^{4-7}$ the tendency of results are qualitatively consistent with the theory, as already examined by Fredrickson. ${ }^{8}$ As reported previously, ${ }^{10,12}$ flow-induced alignment of lamellae was not observed for low molecular weight DP and SP samples. From above discussions, it can be pointed out that the tested shear rates up to $1000 \mathrm{~s}^{-1}$ for those low molecular weight samples are not high enough to cause flowinduced alignment. In other words, the frequency of fluctuations which depends on viscosity of samples and viscosity ratio of components are faster than tested shear rates.

\section{CONCLUSIONS}

We studied flow effects on the structure of lamellar forming poly(styrene- $d_{8}$-block-isoprene)s (DI) solutions under steady shear flow by small angle neutron scattering (flow-SANS) and compared with the results for poly(styrene- $d_{8}$-block-2-vinylpyridine)s (DP), based on the experimentally determined interaction parameters, $(\chi N)_{\text {eff }}$. For DI solutions, it is observed that parallel and perpendicular alignments of lamellar structures coexist at low shear rates and the latter become dominant at high shear rates in the quiescent ordered states and disordered states very close to the quiescent ODT. At still lower concentration below the quiescent ODT, flow-induced ordering with perpendicular alignment is observed. With further decrease of concentration, only slight anisotropy of fluctuation is observed. For DP solutions, coexistence of parallel and perpendicular alignments are only observed at low shear rates for relatively high concentration above quiescent ODT, while only perpendicular alignment was observed for lower concentrations near the ODT. Flow-induced ordering was not observed. The degree of perpendicular alignments (ratio of sector averaged intensities) are compared at similar $(\chi N)_{\text {eff }}$ values and concluded that the degree of alignment is primarily determined by interaction parameters and reduced shear rates. In addition, parallel alignment becomes more stable for the diblock copolymers consist of components with different viscosities in wider ranges of concentration and shear rates.

Acknowledgment. This work was partly supported by a Grant-in-Aid for Scientific Research on Priority Areas (A) (\#13031041-00) from Ministry of Education, Culture, Sports, Science and Technology.

\section{REFERENCES}

1. H. Watanabe, in "Structure and Properties of Multiphase Polymeric Materials,” T. Araki, T. C. Qui, and M. Shibayama, Ed., Marcel Dekker, New York, 1998, chapt. 9.

2. I. W. Hamley, Curr. Opin. Collid Interface Sci., 5, 342 (2000).

3. K. A. Koppi, M. Tirrell, and F. S. Bates, Phys. Rev. Lett., 70, 1449 (1993).

4. K. I. Winey, S. S. Patel, R. G. Larson, and H. Watanabe, Macromolecules, 26, 2542 (1993).

5. K. I. Winey, S. S. Patel, R. G. Larson, and H. Watanabe, Macromolecules, 26, 4373 (1993).

6. N. P. Balsara, B. Hammouda, P. K. Kesani, S. V. Jonnalagadda, and G. C. Straty, Macromolecules, 27, 2566 (1994).

7. N. P. Balsara, H. Dai, P. K. Kesani, B. A. Garetz, and B. Hammouda, Macromolecules, 27, 7406 (1994).

8. G. H. Fredrickson, J. Rheol., 38, 1045 (1994).

9. Y. Takahashi, M. Noda, N. Ochiai, and I. Noda, Polymer, 37, 5943 (1996).

10. S. Kitade, N. Ochiai, Y. Takahashi, I. Noda, Y. Matsushita, A. Karim, A. I. Nakatani, H. Kim, and C. C. Han, Macromolecules, 31, 8083 (1998).

11. Y. Takahashi, M. Noda, S. Kitade, and I. Noda, J. Phys. Chem. Solids, 60, 1343 (1999).

12. Y. Takahashi, M. Noda, S. Kitade, K. Matsuoka, Y. Matsushita, and I. Noda, submitted to Polym. J.

13. Y. Takahashi, N. Ochiai, Y. Matsushita, and I. Noda, Polym. J., 28, 1065 (1996).

14. A. Takano, O. Kadoi, K. Hirahara, S. Kawahara, Y. Isono, J. Suzuki, and Y. Matsushita, Macromolecules, 36, 3045 (2003).

15. Y. Takahashi, S. Kitade, M. Noda, N. Ochiai, I. Noda, M. Imai, and Y. Matsushita, Polym. J., 30, 388 (1998).

16. Y. Takahashi, M. Noda, M. Naruse, T. Kanaya, H. Watanabe, T. Kato, M. Imai, and Y. Matsushita, Nihon Reoroji Gakkaishi, 28, 187 (2000).

17. D. Ehlich, M. Takenaka, and T. Hashimoto, Macromolecules, 26, 492 (1993).

18. L. Leibler, Macromolecules, 13, 1602 (1980).

19. T. P. Lodge, C. Pan, X. Jin, Z. Liu, J. Zhao, W. W. Maurer, and F. S. Bates, J. Polym. Sci., Part B: Polym. Phys., 33, 2289 (1995). 